\title{
Migration um zu überleben: Die spezielle ökonomische Anpassung der Kaiapó-Indianer in Zentralbrasilien 1
}

Immer mehr Anthropologen und Biologen neigen dazu, die Probleme zu betonen, die im Zusammenhang mit dem Leben im Amazonasbecken entstehen, einem Gebiet, das durch ein sehr zerbrechliches ökologisches Gleichgewicht charakterisiert ist. Jüngste Untersuchungen unterstreichen die Vorteile von Siedlungen entlang größeren Flüssen, wo das Land sich fruchtbarer für den Anbau erweist und auch das Wild reichlicher vorhanden ist als in den interfluvialen Gebieten; denn diese tropischen Waldzonen abseits von bedeutenden Flüssen weisen spärliche Wildbestände und ein limitiertes Fischfangpotential auf.

Für solche Siedlungen in interfluvialen Gebieten würde demnach das Hauptproblem darin bestehen, genügend tierische Proteinressourcen in der Nahrung sicherzustellen, wenn eine größere Dorfbevölkerung versorgt werden müßte. Allerdings ist es eine Tatsache, daß die meisten indianischen Dörfer in diesen interfluvialen Gebieten nur eine Bevölkerungszahl von durchschnittlich 80 Einwohnern aufweisen (normalerweise schwankt die Zahl zwischen 30 und 150).

Der vorliegende Artikel behandelt die Ge-sprechenden Kaiapó-Indianer in Zentralbrasilien. Obwohl ihr Siedlungsgebiet in der Zeit vor dem Kontakt mit der Außenwelt noch beträchtlich größer war, umfaßt es heute noch eine Fläche, die ungefähr derjenigen Portugals entspricht. Es erstreckt sich über einen wesentlichen Teil des Südostens von Pará und setzt sich bis in den äußersten Nordosten des Mato Grosso fort. Es sind etwa 3000 Kaiapó, die hier verstreut in dreizehn Gemeinschaften leben, wovon die meisten erst in den 50er und 60er Jahren unseres Jahrhunderts «entdeckt» wurden.

Das Volk der Kaiapó ist ein interessantes Beispiel für die bereits diskutierte Anpassung an eine zerbrechliche ökologische Umwelt, wie sie das Amazonasbecken aufweist, und für eventuell auftretende Proteinknappheit. Denn die Kaiapó sind kein Flußvolk: ihre Dörfer sind traditionell in den interfluvialen Gebieten gelegen (genauer an den Oberläufen von Nebenflüssen oder kleinen Flüssen). Ihre Dörfer weisen jedoch eine überdurchschnittlich hohe Bevölkerung auf im Vergleich zum Amazonasstandard. Zudem basiert die Wirtschaft der Kaiapó auf der Jagd, dem Brandrodungsfeldbau und zu einem geringeren Ausmaß dem Sammeln wilder Früchte und dem Fischfang. Ich möchte mich in diesem Beitrag nicht mit der Frage beschäftigen, wie es zu diesen hohen Bevölkerungszahlen kam, sondern eher damit, wie sie es schafften, solche großen Dörfer in den interfluvialen Gebieten zu versorgen. Doch bevor ich mich diesem Thema zuwende, betrachte ich es als notwendig, die spezielle demographische $\mathrm{Si}$ tuation der Kaiapó zu skizzieren und eine kurze Beschreibung ihrer Wirtschaftslage zu geben.

\section{Dörfer mit über 3000 Indianern}

In Zentralbrasilien ist das Jahr durch zwei Jahreszeiten gekennzeichnet: die Trockenzeit oder der «Winter» von Mai bis Oktober und die Regenzeit oder der «Sommer» von November bis April. Die Trokkenzeit ist charakterisiert durch heiße, windige Tage, kühle Nächte und das fast gänzliche Ausbleiben von Regen. Die Regenzeit hingegen ist durch starke Regenfälle und Überschwemmungen der meisten großen und kleineren Flüsse gekennzeichnet. Die jährliche Regenmenge ist sehr hoch: sie beträgt zwischen $1900 \mathrm{~mm}$ im nordöstlichen Teil des Kaiapó-Gebietes und $2500 \mathrm{~mm}$ im südwestlichen Teil. Die Durchschnittstemperatur erreicht etwa $25-29^{\circ} \mathrm{C}$.

Zu Beginn der Trockenzeit verbringen die Männer die meiste Zeit damit, Bäume zu fällen, um den notwendigen Platz für neue Gärten zu schaffen. Nach dieser Aufgabe ist es nicht unüblich, daß kleine Gruppen sich entfernen und einige Wochen im Wald verbringen. Am Ende der Trockenzeit vereinigt sich die Dorfbevölkerung wieder und setzt ihre Arbeit in den Gärten fort.

Die Durchführung wichtiger Zeremonien charakterisiert die Regenzeit. Jede dieser Zeremonien kann einige Wochen oder gar Monate dauern, und sie ist normalerweise begleitet von größeren Wanderungen in den Wald. Solche Wanderungen zielen darauf

Gustaaf Verswijver, Dr., Musée d'Ethnographie, Boulevard Carl-Vogt 65-67, 1205 Genève 


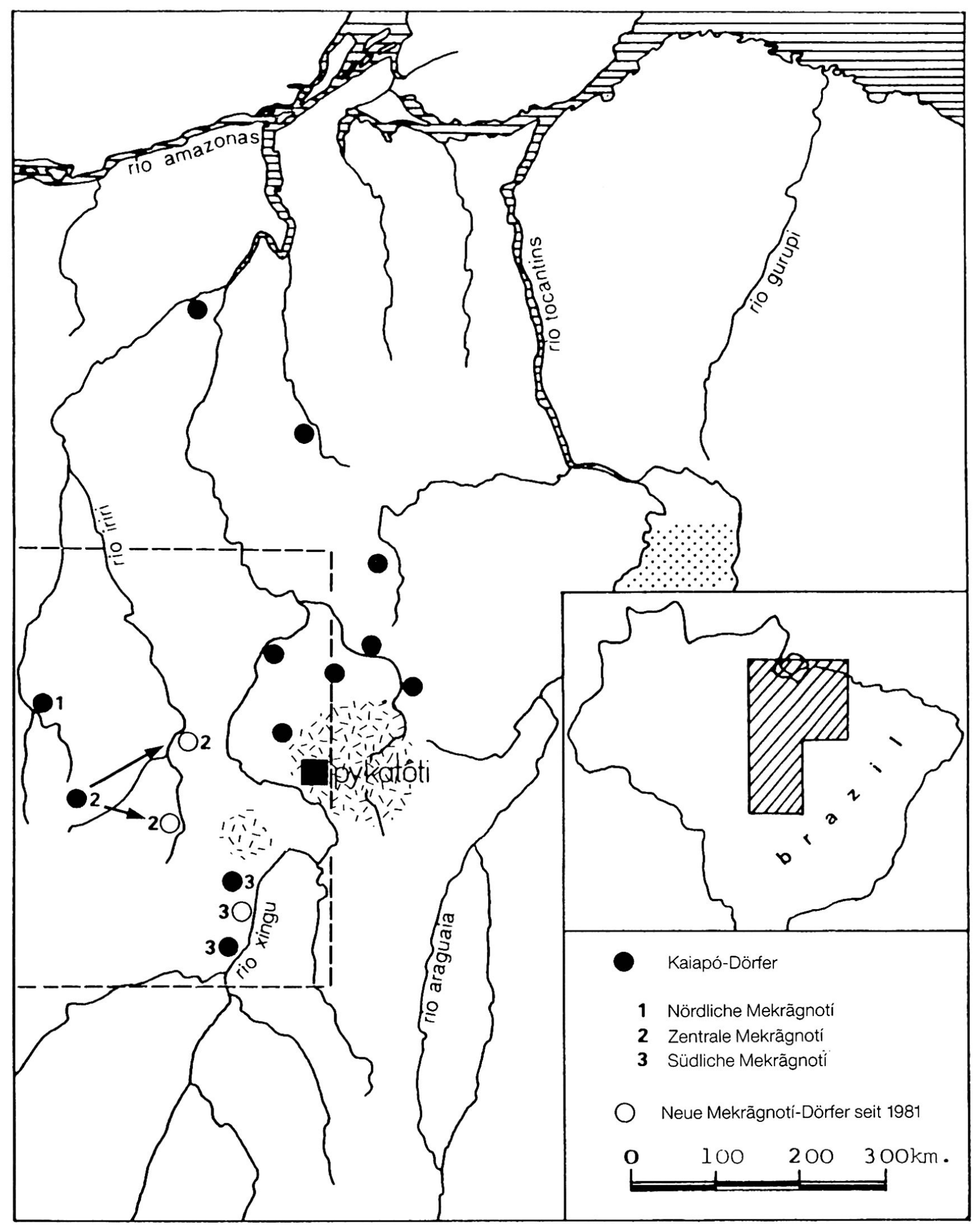

Abb. 1 Karte der geographischen Lage der Mekrãgnoti und anderer Kaiapó-Dörfer (Situation im Jahre 1984)

㴧:- Ungefähre Grenzen vom Gebiet des offenen Graslandes, westlich des Flusses Araguaia

$\because \because \quad \quad$ Gebiet, welches von den Kaiapó im 19. Jahrhundert bewohnt wurde

- - Ausschnitt vergrößert auf Abb. 3 


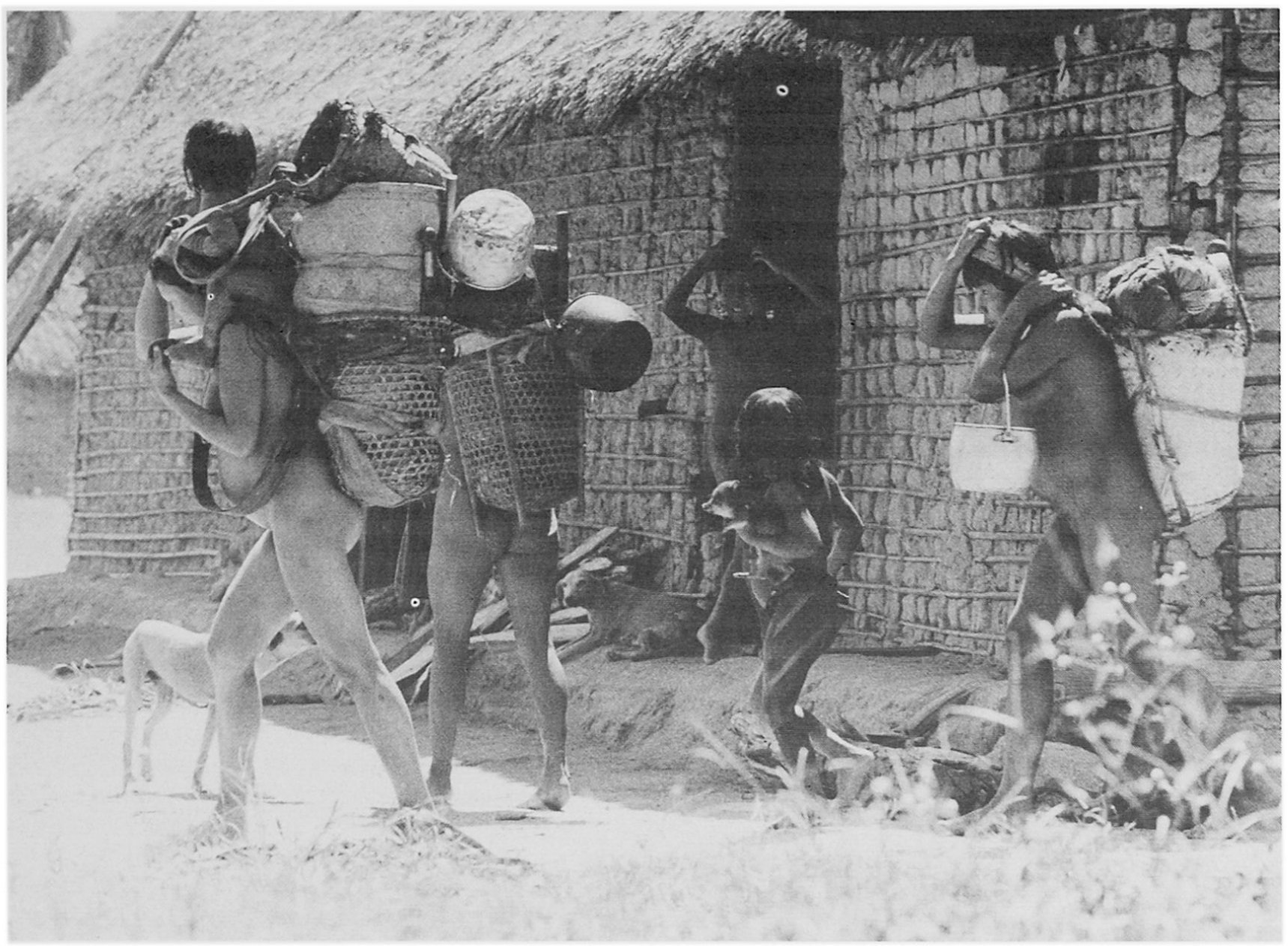

Abb. 2 Frauen, die schwer beladen von einer Waldwanderung zurückkehren, die einen Monat dauerte (Zentral-Mekrãgnoti). Photo: G. Verswijver, 1976.

ab, das notwendige Wild (oder gelegentlich Fisch) für die Schlußphase der Zeremonie zu besorgen.

$\mathrm{Zu}$ Beginn des 19. Jahrhunderts besiedelten die Kaiapó ausgedehnte Gebiete zwischen der unteren Araguaia und dem oberen Tocantins, ein Gebiet, das mit offenem Grasland und Galeriewäldern entlang den Wasserläufen bedeckt ist. Durch die sich ausbreitende Kolonialisierung wurden die Kaiapó mehr und mehr verdrängt und dazu gezwungen, weiter ins Landesinnere zu ziehen. Die Hauptwanderung fand im Verlauf des 19. Jahrhunderts statt und zwang die Kaiapó zur Besiedlung eines Gebietes, das eine Übergangszone zwischen offenem Grasland und tropischem Regenwald darstellt. Einige Gruppen, so die Zentral-Mekrãgnotí ${ }^{\text {, }}$ migrierten noch weiter westwärts und ließen sich schließlich in einem von dichtem tropischem Regenwald bedeckten Gebiet nieder (siehe Karte, Abb. 1). Bedeutende Dörfer wurden normalerweise im offenen Grasland, nahe der Grenze zum tropischen Regenwald, gebaut. In einer solchen Übergangszone zu leben, erlaubte es den Kaiapó, die natürlichen Ressourcen beider ökologischen Zonen auszunutzen.

Ethnohistorische Untersuchungen zeigen, daß alle dreizehn heutigen Kaiapó-Dörfer von einer einzigen Ahnengruppe abstammen, welche sich im 19. Jahrhundert auf drei Hauptdörfer aufteilte. Später splitterte sich jedes dieser drei Dörfer in kleinere Gemeinschaften auf (VERSWIJVER, 1985).

Als sich mit den Brasilianern in den 50er und 60er Jahren die ersten dauerhaften friedlichen Kontakte ergaben, waren die Kaiapó über eine große Zahl von Dörfern verstreut, welche alle eine Bevölkerungszahl von 200 bis 800 Indianern aufwiesen. Doch zeugen historische Dokumente davon, daß man in einigen damaligen Dörfern noch weitaus mehr Einwohner zählte. Es wird allgemein angenommen, daß die großen Kaiapó-Dörfer im ausgehenden 19. Jahrhundert über 1000 Einwohner aufwiesen.

Diese Schätzungen stimmen etwa überein mit denen von Pykatôti, einem Kaiapó-Dorf im offenen 
Grasland östlich des Riozinho-Oberlaufes gelegen (siehe Karte, Abb. 1). Pykatôti wurde von etwa 1870 (oder früher) bis 1935 bewohnt. Posey, der die betreffende archäologische Stelle 1979 besuchte, schätzte den Durchmesser des Kreises, der von den Wohnhäusern gebildet wurde ${ }^{3}$, auf 1050 Meter (!) und schlo $\beta$ daraus, da $\beta$ zur damaligen Zeit etwa 3700 bis 4500 Indianer dort gelebt haben müssen (POSEY, 1979: 56). Im Laufe dieses Jahrhunderts teilten sich die Pykatôti in mehrere Gruppen auf, von denen sich jede später in mehrere Dörfer aufsplitterte. Elf von den dreizehn heutigen Kaiapó-Dörfern stammen von den Pykatôti ab.

Um ungefähr 1905 trennten sich etwa 250 Indianer von den Pykatôti ab. Sie nahmen den Namen Mekrãgnotí («das Volk mit der tiefroten Gesichtsfarbe») an und migrierten weiter westlich, wo sie sich schließlich zwischen dem Xingu- und Iriri-Fluß niederließen. In den folgenden Jahrzehnten wuchs die Bevölkerung der Mekrãgnotí in schnellem und stetigem Ausmaß. 1937 zählte ihr Dorf 650 Einwohner, einschließlich 120 abtrünniger Pykatôti-Indianer, die eben neu dazugekommen waren; sie bewohnten alle ein einziges Dorf. Ethnohistorische Untersuchungen deuten jedoch darauf hin, daß von 1945-47 die Mekrãgnotí-Dörfer zwischen 750 und 800 Indianer zählten. Kurz darauf begann sich die Gruppe aufzuteilen, und zur Zeit, da sie mit den Brasilianern in friedlichen Kontakt traten (in der Periode zwischen 1953 bis 1957), hatte die gesamte Mekrãgnotí-Bevölkerung eine Zahl zwischen 900 und 1000 Indianern erreicht, die in drei Dörfern verteilt lebten.

Wir werden nun sehen, wie andauernde Kontakte mit unserer Gesellschaft die Subsistenz-Aktivitäten sowie die Migrationsstrategie der Kaiapó verändert haben.

\section{Leben an einem interfluvialen Standort}

Signifikante Änderungen in den Subsistenz-Aktivitäten der Mekrãgnotí in jüngster Zeit, wie z. B. die wachsende Abhängigkeit vom Fischfang als Proteinressource oder weniger häufige Waldwanderungen, können im Grunde einem einzigen Phänomen zugeschrieben werden: der seßhaften Lebensweise, die erst in letzter Zeit den Lebensraum der Gruppe zu beeinflussen begann.

Vor der sogenannten "Befriedung» in den Jahren 1953-1957 bewohnten die Mekrãgnotí ausgedehnte interfluviale Gebiete in Zentralbrasilien. Zahlreiche Dörfer waren zwischen den Flüssen Xingu und Jamanxim (siehe Karte, Abb. 3) gebaut worden, jedoch wurden diese über das Stammesgebiet verstreuten Dörfer oft nicht länger als ein oder zwei Jahre bewohnt. Die Bevölkerung tendierte viel- mehr dazu, ständig von einem Dorf zum anderen zu ziehen. Solche Wanderungen, bei denen oft relativ große Distanzen zurückgelegt wurden (gelegentlich über $150 \mathrm{~km}$ ), hatten wichtige wirtschaftliche Gründe und Folgen. Im jeweiligen Dorf ernteten sie vorerst aus den Gärten, die bei einem früheren Aufenthalt angelegt worden waren, pflanzten jedoch auch jedes Mal neue Anbauflächen an. Dieses Vorgehen, ständig in früher bewohnte Dörfer zurückzukehren, sicherte eine ständige Regenerierung der verfügbaren Gartenflächen, verteilt über praktisch das ganze Stammesgebiet.

Dieses dauernde Umherziehen ist zweifellos mit dem zeremoniellen Leben verbunden. Die meisten Rituale, wie z. B. die Zeremonie der Namensgebung, sind gekennzeichnet durch ein festes Muster von vier Phasen:

1. Die meist lange Vorbereitungsphase, während welcher Lieder gelehrt und geübt werden;

2. die zeremonielle Wanderung, wobei große Mengen von Fleisch und Fisch erbeutet werden. Dies geschieht entweder dadurch, daß man Hunderte von Schildkröten sammelte oder Herden wilder Schweine jagte oder die «timbo»-Fischfangtechnik anwandte ${ }^{4}$;

3. die Zubereitung der Nahrung, nicht nur aus Fleisch und Fisch, sondern auch aus großen Mengen von Maniok und Maismehl;

4. der Höhepunkt der Zeremonie, bei der die ganze Nacht getanzt wird und der größte Teil der zubereiteten Nahrung verspiesen wird.

Wenn nicht rein zufällig in der gegebenen Zeit eine Herde wilder Schweine auftauchte, war man in der zweiten Zeremoniephase gezwungen, eine zeremonielle Wanderung zu unternehmen. Solche Wanderungen führten entweder zu einem größeren Fluß zum Fischfang oder dann in den Wald, um Schildkröten zu sammeln. Die dritte Phase wiederum erforderte große Mengen an Maniok und Mais von den Gärten des Namensgebers.

Es müssen hierbei zwei Arten von zeremoniellen Wanderungen unterschieden werden:

a) Die erste bezeichne ich als «zirkuläre Wanderung», bei welcher die Leute das Dorf verlassen und eine kreisförmige Wanderung um eine Seite der Siedlung machen und schließlich in dieses Dorf zurückkehren (siehe Abb. 4);

b) die zweite würde ich als «lineare Wanderung» bezeichnen, bei welcher die Leute das Dorf verlassen und langsam zu einem andern Dorf wandern, wobei sie dort die früher angelegten Gärten nutzen und so die erforderlichen Mengen an Maniok und Mais für Phase 3 resp. 4 einbringen können.

Die Entscheidung für die eine oder andere Art der Wanderung hing von verschiedenen Faktoren ab, wie z. B. den verfügbaren Mengen an Maniok und Mais; der Zeitdauer, während welcher ein Dorf be- 


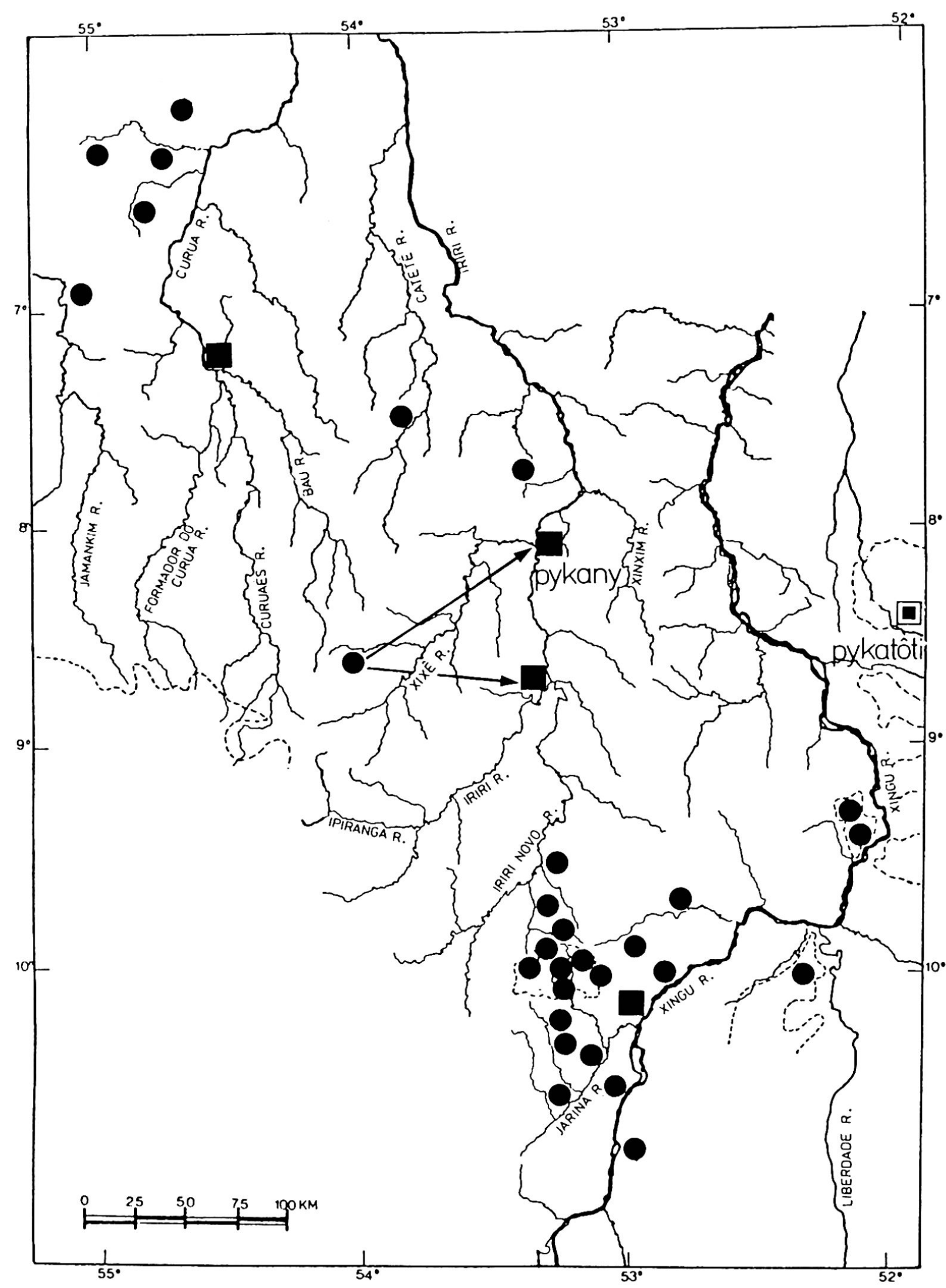

Abb. 3 Die Lage aller wichtigen Mekrãgnoti-Dörfer im 20. Jahrhundert (1905-1984)

- heutige Mekrãgnoti-Dörfer

- verlassene Mekrãgnotí-Dörfer

-. Grenze des offenen Graslandes 


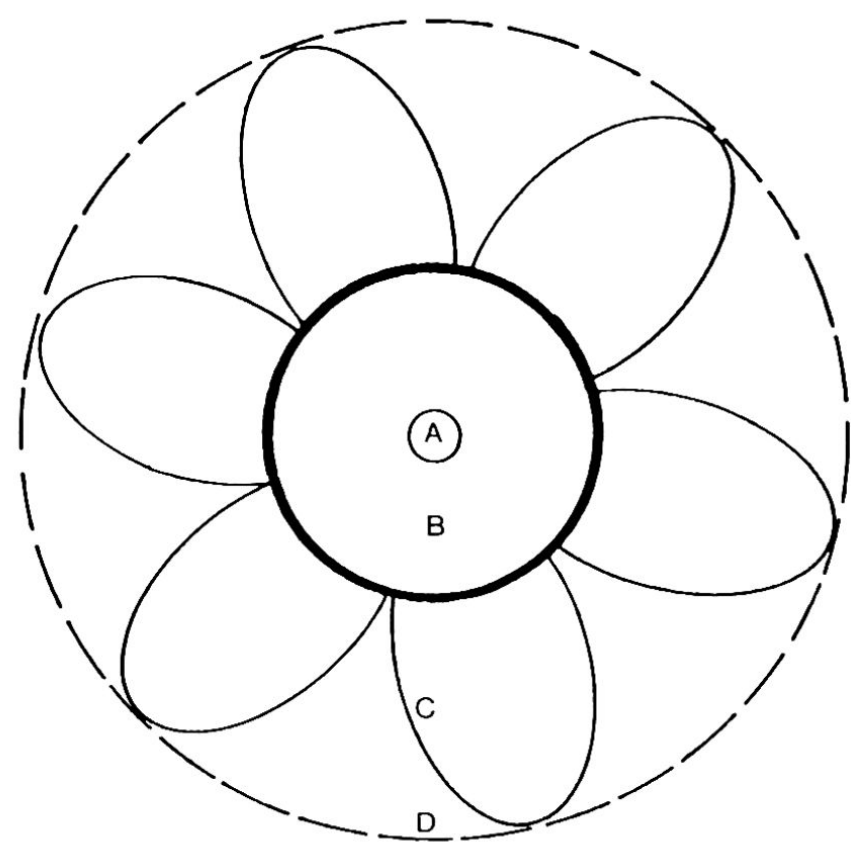

Abb. 4 Diagramm, welches ein Modell der Ausbeutung der natürlichen Ressourcen um das Dorf zeigt, indem abwechslungsweise zirkuläre Wanderungen in verschiedene Richtungen unternommen werden.

$A=$ Dorf; $B=$ Gebiet der täglichen Jagd; $C=$ Routen der zirkulären Wanderungen; $D=$ Grenze des ausgebeuteten $\mathrm{Ge}$ bietes, währenddem ein Dorf bewohnt wird.

wohnt wurde; und der Richtung der Wanderung. Es schien eine Tendenz vorhanden zu sein, das erste oder auch das zweite Jahr die «zirkuläre» Wanderung beidseits des Ausgangsdorfes zu wählen (siehe Abb. 4) und dann im zweiten resp. dritten Jahr zur «linearen» Wanderung überzugehen. Dabei griff man wie bereits erwähnt auf die früher angelegten Gärten zurück ${ }^{5}$.

Die meisten Migrationen von einem Dorf in ein anderes wurden daher als «lineare Wanderung» unternommen und fielen somit mit dem Zyklus der Rituale zusammen. Doch scheint es einige Basisdörfer gegeben zu haben, die sich alle im offenen Grasland zwischen den Flüssen Jarina und Iriri Novo befanden, in welche die Indianer normalerweise zurückkehrten, nachdem sie zwei bis fünf Jahre in anderen benachbarten Dörfern verbracht hatten. Abb. 5 zeigt ein Modell dieses komplexen traditionellen Wanderungsmusters der Mekrãgnotí. Dieses ständige Umherziehen der Mekrãgnotí-Gruppen gemäss dem Modell führte zu folgenden Gegebenheiten:

1. Die Kontrolle über ihr Stammesgebiet wurde verbessert;

2. für den Fall, daß sie angegriffen wurden, hatten sie immer Dörfer, in die sie fliehen konnten und deren bereits angelegte Gärten ihnen das Weiterleben im gleichen Stil zusicherten;

3. der Proteinanteil der Nahrung erhöhte sich.

Dieser letzte Punkt wurde vor allem durch die regelmäßigen Wanderungen zwischen dem offenen Grasland und dem tropischen Urwald erreicht. Denn dadurch konnten nicht nur Ressourcen von verschiedenen Gebieten, sondern auch von verschiedenen ökologischen Zonen genutzt werden. Die Gefahr, an den ökologisch anfälligeren Ressourcen der interfluvialen Gebiete Raubbau zu betreiben, wurde auf diese Weise vermindert ${ }^{6}$.

\section{Jüngste Veränderungen}

Wie schon erwähnt, sind im Gegensatz zu diesen traditionellen Wanderungsprozessen, wie sie vor dem Kontakt mit der Außenwelt bestanden, mehrere heutige Kaiapó-Dörfer schon seit zwanzig oder dreiBig Jahren nicht mehr verlassen worden. Die Wanderungen, die noch immer häufig unternommen werden, beschränken sich heute auf die nähere Umgebung und sind damit fast ausnahmslos vom «zirkulären» Typ. Die Wanderungsaktivitäten spielen sich somit in der nächsten Umgebung eines ständig bewohnten Dorfes ab.

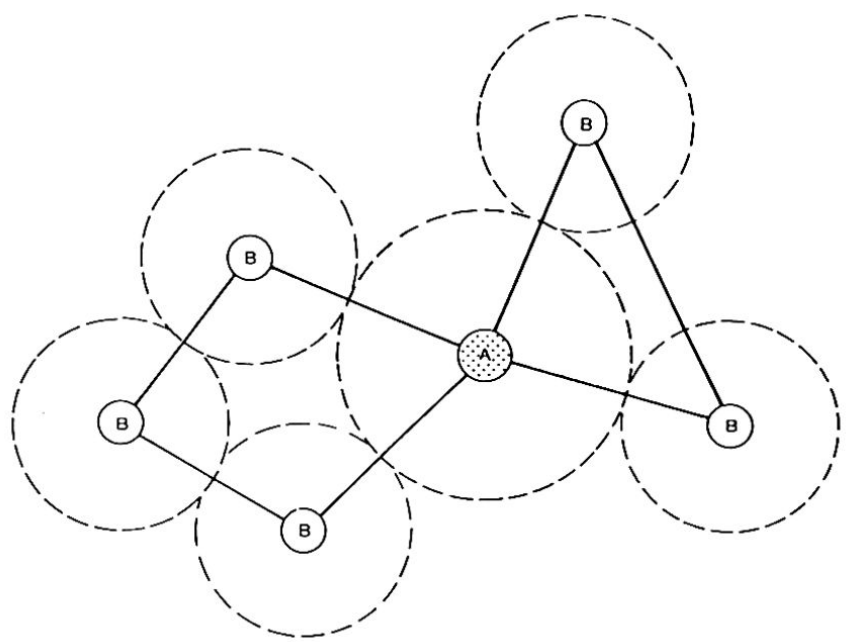

Abb. 5 Modell des Wanderungsmusters der Mekrãgnotí in der Zeit vor dem Kontakt mit der Außenwelt.

$A=\quad$ Hauptdorf, zu welchem die Indianer öfters zurückkehren

$\mathrm{B}=\quad$ Provisorische Dörfer, die abwechslungsweise bewohnt sind, wenn das Hauptdorf verlassen wird

- - - Grenzen des Gebietes, welches bearbeitet wurde nach mehreren "zirkulären Wanderungen" (siehe Abb. 4)

Lineare Wanderungen nach provisorischen Dörfern 
Zwei wichtige Veränderungen haben sich hieraus ergeben:

a) Die heutige seßhafte Lebensweise im Dorf;

b) die Begrenzung der Wanderungsmuster.

Beide Veränderungen müssen zudem mit den Niederlassungen von Missions- und Regierungsstellen in Verbindung gebracht werden. Diese Stellen verlangen eine gewisse Infrastruktur, wie ständig bewohnte Häuser, einen kleinen Fluglandeplatz, die Installation von Radios und die Abgabe von Medikamenten. Sie können nicht einfach verschoben werden. Diese Infrastruktur ist auch ein Anziehungspunkt für die Indianer (erhältliche Handelsgüter, medizinische Versorgung, Radio und Besucher, die über andere Dörfer informieren), und damit werden heutige Kaiapó-Dörfer kaum mehr verlegt.

Die meisten dieser Niederlassungen wurden zwischen 1940 und 1960 gebaut, und zwar immer an einem größeren Fluß, um den Wassertransport zu erleichtern. Diese Konzentration von heutigen Kaiapó-Dörfern an großen Flüssen hat zur Folge, daß die erforderlichen Mengen an Fleisch und Fisch für die Zeremonien nicht mehr zwingend lange Wanderungen erfordern (solche Wanderungen finden zwar immer noch statt, aber viel weniger häufig als in den Zeiten vor der Kontaktnahme), sondern durch Fischfang neben dem Dorfareal gedeckt werden können.

Eines der letzten Kaiapó-Dörfer, welches sich noch an einem rein interfluvialen Standort befand, ist dasjenige der Zentral-Mekrãgnotí in der Nähe von P. I. Mekrãgnotí?. Diese Ausnahme kann jedoch durch die historische Entwicklung der «Befriedung» von 1953-1961 erklärt werden. Als die Indianer 1953 zum ersten Mal mit der Außenwelt in Kontakt kamen, wurden sie von den im Dorf auftretenden Krankheiten so verängstigt, daß mehr als 500 Zentral-Mekrãgnotí das Gebiet um den Jarina-Fluß verließen und weiter landeinwärts ein neues Dorf gründeten. Zwischen 1957 und 1961 hat die Regierungsbehörde nacheinander drei Niederlassungen am Iriri- und Curuães-Fluß eröffnet, mit der Hoffnung, damit die Indianer entlang einem größeren Fluß anzusiedeln.

Doch jedes Mal verließen die Zentral-Mekrãgnotí diese Stellen, nachdem sie nach einigen Monaten wegen auftretender Krankheiten arg dezimiert worden waren. In der Mitte der 60er Jahre begannen die Missions- und Regierungsinstitutionen Lufttransporte zu organisieren, um auch die isolierten Niederlassungen zu versorgen.

1967 wurde eine Niederlassung in der Nähe des interfluvialen Standorts des Mekrãgnotí-Dorfes gebaut. $\mathrm{Zu}$ diesem Zeitpunkt betrug die Bevölkerungszahl lediglich noch 137 Indianer.

Während die Zentral-Mekrãgnotí in der Zeit von 1953-1967 noch öfter in andere Dörfer umgezogen

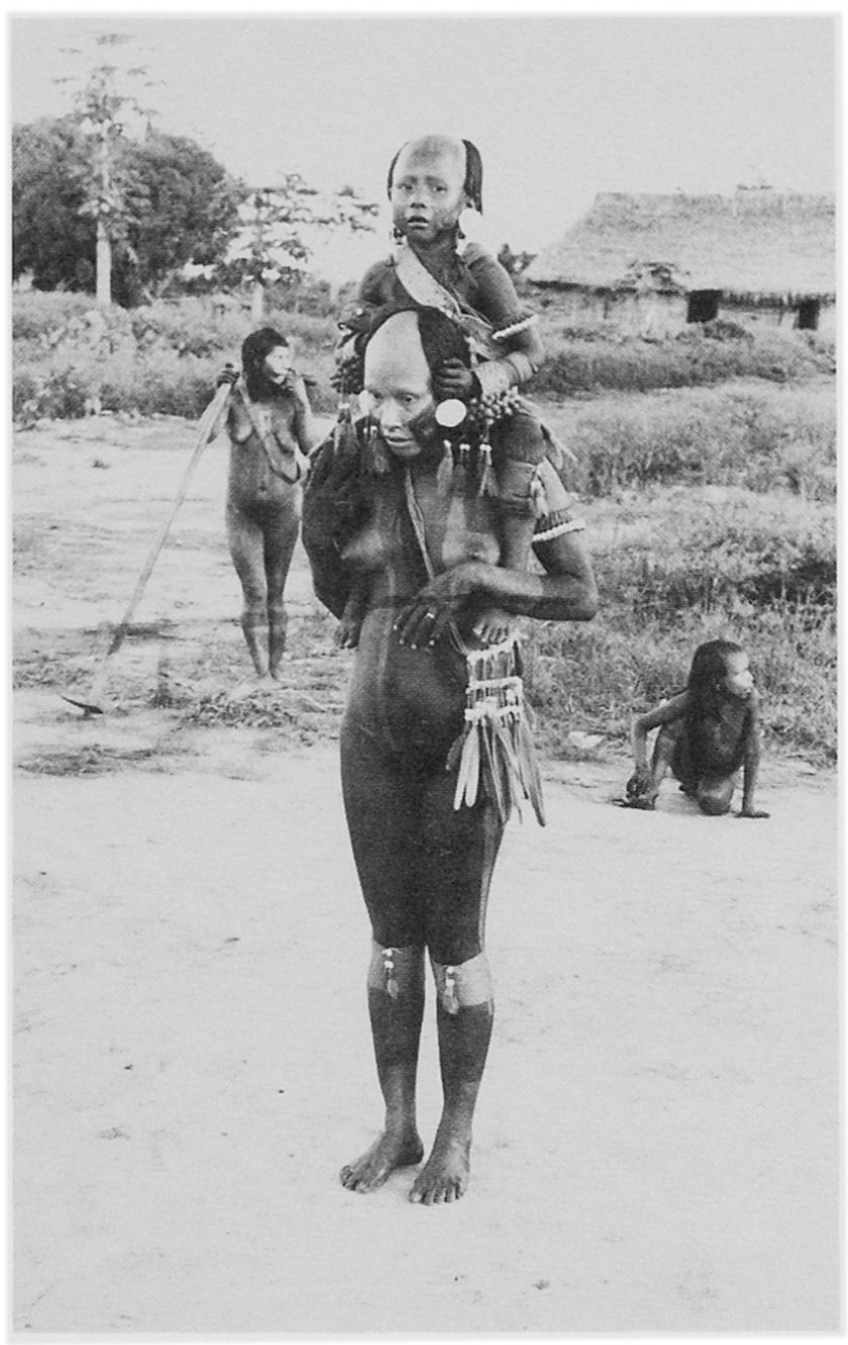

Abb. 6 Geschmücktes Kind, das während einer wichtigen Zeremonie von seiner Tante getragen wird (Zentral-Mekrãgnoti).

Photo: G. Verswijver, 1978.

waren, führten sie ab 1967 ein seßhaftes Dorfleben. Die meisten zeremoniellen und saisonalen Wanderungen, welche die Mekrãgnotí noch unternahmen, waren vom «zirkulären» Typ, mit Ausnahme von einigen saisonalen Wanderungen, welche die Gruppe vorübergehend ins nördliche MekrãgnotíDorf in P. I. Bau führte.

Doch das seßhafte Dorfleben im interfluvialen Gebiet führte zu einem allmählichen Raubbau an verfügbarem Fleisch. Es mußten immer häufiger Fischfang-Wanderungen zum Xixê-Fluß (etwa $40 \mathrm{~km}$ östlich des Dorfes) unternommen werden. Auf halbem Wege zum Fluß und entlang dem Fluß wurden Gärten angelegt, um die reisenden Familien und Wanderungsgruppen zu versorgen. Es wurden oft mehrere saisonale Wanderungen zu diesen Punkten durchgeführt. 
Nachdem die Zentral-Mekrãgnotí mit einer stetig wachsenden Bevölkerungszahl über zehn Jahre am gleichen Ort gelebt hatten, wurde der Proteinmangel zu einem Problem. Als ich dieses Dorf 1974 zum ersten Mal besuchte (zu dieser Zeit lag es immer noch am gleichen Ort), zählte die Bevölkerung 254 Indianer, und die Zuwachsrate betrug 7\%! Um 1978 traten ernsthafte wirtschaftliche Probleme auf. Die Knappheit an Wild in der Nähe des Dorfes verschlimmerte sich durch den Umstand, daß $65 \%$ der Dorfbevölkerung jünger als zwölf Jahre war. Diejenigen Paare mit vielen Kindern begannen sich zu beklagen, daß ihre Kinder kein Fleisch zu essen bekämen. Diese Familien brachten auch die Idee auf, das Dorf in die Nähe eines größeren Flusses zu verschieben. Die meisten Dorfälteren waren aber gegen diesen Plan. Sie argumentierten, da $\beta$ die Leute eher an Krankheiten leiden würden, wenn sie entlang einem großen Fluß lebten (so wie sie es selbst von 1953 bis 1961 erlebt hatten). Schließlich entschied die Mehrheit der Bevölkerung, im bestehenden Dorf zu bleiben. 1981 machten sich jedoch etwa achtzehn Männer auf, um ein neues Dorf entlang dem Fluß Iriri aufzubauen. Die Niederlassung in der Nähe dieses neuen Dorfes wurde P. I. Pykany genannt.

Die begeisternden Erzählungen von vorhandenem Fisch und Fleisch bei einem Besuch der Ausgewanderten im alten Dorf überzeugten die verbliebene Hauptgruppe, ein neues Dorf am Fluß zu bauen. Die Stelle am Iriri Novo, flußaufwärts von P. I. Pykany, wurde so gewählt, daß die Erwartungen beider Gruppen, der Leute von P. I. Pykany wie auch von P. I. Mekrãgnotí, erfüllt werden konnten. Nämlich:

1) Das Dorf liegt nahe am Fluß, der sehr fischreich ist (vor allem die Pykany-Leute waren damit zufrieden);

2) der Iriri-Novo-Fluß ist ein relativ kleiner Wasserlauf und befriedigte damit die Wünsche der Dorfälteren, welche Angst hatten, an einem großen Strom zu leben. Außerdem liegt dieser neue Dorfplatz näher beim traditionellen offenen Grasland, wo die meisten Dorfälteren geboren wurden.

\section{Schlußfolgerung}

In diesem Artikel habe ich gezeigt, wie es die Mekrãgnotí erreicht haben, in traditioneller Weise an einem interfluvialen Standort wirtschaftlich zu überleben, und dies trotz hohen Bevölkerungszahlen. Vor der sogenannten «Befriedung» in den Jahren 1953-1957 wurden die meisten MekrãgnotíDörfer im offenen Grasland gebaut, am Rande der Galeriewälder oder in der nahen Umgebung des tro- pischen Regenwaldes. Indem sie Wanderungen von einem Dorf ins andere unternahmen, wurde die Ausbeutung des Wildes auf verschiedene Jagdgebiete verteilt. Zudem wurden abwechslungsweise verschiedene ökologische Zonen beansprucht, so $\mathrm{da} ß$ trotz hoher Bevölkerungsdichte - vor dem Kontakt mit der Außenwelt waren Dörfer mit 700 und mehr Indianern keine Seltenheit - kein Raubbau an den Ressourcen auftrat.

Das gleiche Muster von Halb-Nomadismus - oder eigentlich besser Halb-Seßhaftigkeit - wurde wahrscheinlich in den ehemaligen Kaiapó-Dörfern befolgt, welche nach POSEY 1979/56) über 3000 Indianer gezählt haben dürften.

Aus dem Englischen übersetzt von Gary Seitz.

\section{Summary}

In the present paper, it is shown how the Kaiapó Indians of Central Brazil traditionally managed to survive in an ecologically unfavorable habitat, and such while maintaining village populations of no less than 3000 indians. It is also shown how the more or less permanent contacts with our society have affected their traditional pattern of constant movement between a series of villages spread over a huge area.

\section{Anmerkungen}

1 Dieser Artikel stellt einen überarbeiteten Teil des Kapitels 5 meiner Dissertation dar (Nerswijver, 1985). In der Zeit von 1974 bis 1981 verbrachte ich über 33 Monate bei den Kaiapó. Dieses Forschungsprogramm wurde hauptsächlich finanziert durch drei belgische Institutionen (die Fondation Léopold III, die Fondation Nationale de la Recherche scientifique und die Fondation de la Vocation) sowie durch die Fondação de Amparo à Pesquisas (FAPESP) von São Paulo. Ich möchte diesen drei Institutionen danken für die Finanzierung der Feldforschung wie auch der "Brazilian National Indian Foundation" (FUNAl) und der "National Research Foundation" (CNPq) für die Erlaubnis, die Feldforschungen durchzuführen. Ich schulde vor allem Dr. Darrell Posey (Museu Paraénse Emílio Goeldi, Belém) und Ms. Ruth Thomson (Summer Institute of Linguistics, Brasilia) meinen Dank für die freundliche Mitarbeit während der verschiedenen Phasen meines Forschungsprogramms.

2 Mekrãgnotí ist der Name der südlichst gelegenen KaiapóUntergruppe. Es existieren drei Gruppen, welche ich willkürlich als nördliche, zentrale und südliche Mekrãgnoti bezeichne, je nach ihrer geographischen Lage. Heutzutage bewohnt jede dieser drei Gruppen ein oder zwei Dörfer, früher waren es zeitweise mehr. Während den verschiedenen Aufenthalten der Feldarbeit wurden die Untersuchungen in all diesen Dörfern durchgeführt, jedoch lebte ich meist im isolierteren Dorf der Zentral-Mekrãgnoti. 
3 Traditionelle Kaiapó-Dörfer bestehen aus einem kreisförmigen Ring von gleich großen Wohnhäusern, die den Großfamilien Platz bieten und um einen großen, gerodeten Platz herum gebaut werden. Dieser Platz ist das Zentrum eines Kaiapó-Dorfes. Auf ihm steht das Männerhaus, in welchem sich täglich die politischen Männergruppen - «Männergesellschaften" - versammeln. Es stellt damit auch das symbolische Zentrum der komplexen Kaiapó-Gemeinschaften dar, wie übrigens aller $\mathrm{Ge}$-sprechenden Volksstämme.

4 Timbo wird eine Lianenart genannt, die zum Fischfang am Ende der Trockenzeit (August bis Oktober) benützt wird, wenn der Wasserstand am niedrigsten ist.

5 Posey $(1984,114)$ bemerkt hierzu, daß die "neuen Felder» der Kaiapó erst nach zwei bis drei Jahren ihre höchsten Erträge erreichen, jedoch noch weitere Jahre darnach ertragsfähig sind (z. B. Süßkartoffeln während 4-5 Jahren, Yams und Taro 5-6 Jahre, Maniok 4-6 Jahre und Papaya mehr als 5 Jahre). Einige Bananensorten können sogar 15-20 Jahre Früchte tragen, "urucu" während 25 Jahren und "cupa" (cissus gongylodes) gar während 40 Jahren. "Cupa" ist ein wichtiges Grundnahrungsmittel der Kaiapó und außer bei den Indianern wenig bekannt.

${ }^{6}$ Carneiro $(1970,245)$ erwähnte, daß nicht auf den Fischfang, sondern auf die "Jagd abgestellt werden müsse, um den Hauptteil an der Proteinversorgung zu sichern"; und daß
Proteinmangel "von ganz besonderer Bedeutung für ein Siedlungsmuster ist, da eine starke Abhängigkeit von der Jagd mit der seßhaften Lebensweise unvereinbar ist".

7 P.I. ist die Abkürzung von Posto Indigena, die brasilianische Bezeichnung für Regierungsniederlassungen, die in der Nähe von einheimischen Dörfern errichtet wurden.

\section{Literatur:}

CARNEIRO, R. (1970): The Transition from Hunting to Horticulture in the Amazon Basin. Eigth Congress of Anthropological and Ethnological Sciences, Tokyo.

POSEY, D. (1979): Pykatôti: Kaiapó mostra aldeia de origem. In: Revista de Actualidade Indígena, Brasília, vol. 15: 50-57. POSEY, D. (1984): A Preliminary Report on Diversified Management of Tropical Forest by the Kaiapo of the Brazilian Amazon. In: Advances in Economic Botany, vol. 1: 112-126.

VERSWIJVER, G. (1985): Considerations on Mekrãgnotí Warfare. Unveröffentlichte Dissertation der Universität Gent (Belgien). 